\title{
One Stop Center For Disaster Training Information In Smartphone Platform: A Mobile Prototype
}

\author{
http://dx.doi.org/10.3991/ijim.v9i4.4418 \\ Nik Nadian Nisa Nik Nazli, Sapora Sipon and Norita Md Norwawi \\ Universiti Sains Islam Malaysia, Nilai, Negeri Sembilan, Malaysia
}

\begin{abstract}
Smart mobile phones are used in a variety of activities, including social networking, messaging, connectivity and context-sensitive applications. The ubiquities of handheld computer technology have been found to be very useful, especially in flood training applications. The aim of this paper is to propose a prototype for one stop center disaster training information through smart mobile phone application called 'MyDisasterTraining'. Smart mobile phones are used as the medium between the community and organizations in promoting the training provided to the public, as well as providing training services to the community about the importance of disaster training. 'MyDisasterTraining' application is a center database and contains the information about flood disaster training in the Malaysian context. It is a simple, user-friendly, and a low cost application which is a mobile application to be used by the third parties training providers, organizations or individuals. It aims to spread the awareness on the availability of disaster training to the society as well as to inculcate voluntarily workforce during the need of the disaster. The utilization and the roles of ICT are described in the context of disseminating the training information to the society, especially on the training of flood disaster. The features and benefits of the application are also described and explained in this paper
\end{abstract}

Index Terms - Disaster training, Information Communication Technology, Smart mobile phone applications

\section{INTRODUCTION}

Disaster training is one of the trainings that should be promoted to the public servants in Malaysia. Whether public or private sectors, volunteers for this exercise will be helpful in terms of thorough preparation for disasters such as floods. Preparedness is an action taken in advance before an emergency occurs to facilitate a quick and effective response in the event of an emergency. Preparation is important because it can help to save lives, reduce injuries, limit property damage, and reduce the threat of violent disorder that may cause injury [1]. To ensure prompt response and effective disaster management, the local community volunteers have to undergo a basic training, followed by a periodic refresher training, assisted by the Department of Disaster Management [2].

Disaster preparedness must be supported by public education campaigns, training responders, and emergency training scenario. The purpose of the public education and awareness program is to promote information to the society, to be alert and independent, able to play a full role in supporting and working with government officials, and also being responsible for disaster management training [3].

Civilians, aid workers, volunteers, and organizations work together in the disaster preparedness trainings provided by the government or private organizations. Conveying information regarding disaster training to the public is hard as there is no effective approach. One of the ways to disseminate information on disaster training is through information and communications technologies (ICT). It is one of the fastest and effective ways to disseminate information on the training provided by the government or private organizations. Mobile phone is an example of ICT, particularly in the form of mobile devices. In terms of mobile devices, Peters [4] suggested the following three criteria to determine whether a device can be described as a mobile technology:

1. Capable of providing communication and / or information

2. Small enough to be carried easily

3. Can be used (at least part of the time) without a physical connection to a fixed power or telecommunication services

Mobile phone is one of the ICT tools that are popular in relaying information to the public and now the term mobile phone is changed to smart mobile phone $[5][6]$. ElHussein 171 mentioned that the growth of mobile technology is a great opportunity to learn about devices such as smart phones, mobile phones, and tablets. With recent advances in technology that incorporates features that support mobile, digital, and wireless lifestyle, teaching and learning experiences become more interesting. Nowadays, mobile devices such as smart phones are equipped with location information receiver, camera, RFID readers, and other environmental awareness sensors that can provide rich and interactive multimedia content for educational purposes [8].

Smart mobile phone is a medium that replaces television and radio to communicate, deliver, and co-ordinate information quickly to a large number of people. With the advent of mobile apps for smartphones, they can help people to save time and reduce cost, [9][10]. In this paper, we propose a mobile prototype application called MyDisasterTraining. The features and benefits of the mobile application MyDisasterTraining are discussed below. Utilization and roles of ICT are described in the context of disseminating training information to the society, especially regarding trainings for floods. 


\section{The Role of ICT IN Disaster TRAINING INFORMATION}

Information and communication technology (ICT) is a broad term that refers to all computer-based advanced technology to manage and deliver information. The definition is wider than the information technology (IT), which is defined as the study, design, development, implementation, support or management of computer-based information systems, particularly software applications and computer hardware. For relief efforts, communication is referred as the central nervous system [111. Without the coordination, communication is impossible, and lack of communication will cause limited exchange of information. Communication technology enables employees to communicate with the headquarters and also with other

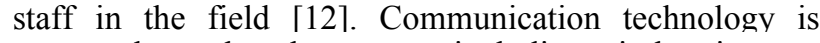
commonly used as the support, including wireless internet connection / satellite TV, mobile phones, satellite phones and radios.

In the ICT domain, the potential of ICT tools regardless of their ultimate goal gives huge impact to the community. Typically, ICT is employed with three measures: (1) for data and information, (2) to transform data and information into knowledge that can be shared and, finally (3) presenting data, information and knowledge (See Figure 1).

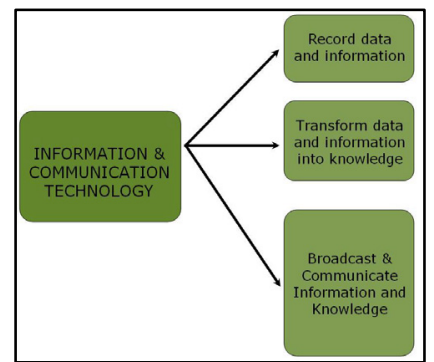

Figure 1. Key Features of ICT

Information and communication technology (ICT) plays an important role in recognizing, managing, supporting and monitoring the community. ICT can help in communication and build trust between the government, community and volunteer. The use of ICT such as smart mobile phones can help to improve dissemination of information [1], such as information on disaster training and building trust between networks, contributes to the effectiveness of community support networks and access to related resources. This will also support and strengthen the organization's role in providing a range of training and community disaster volunteers.

Smart mobile phone application is a platform that provides a new channel to the community to voice their opinions, views and concerns. It allows interaction with an organization, which is an example of the potential of ICT for the transparency and clarity in disseminating information because it is an important factor for perceptions, expectations and beliefs of the local community. Smartphone has proven to be an effective and efficient tool to facilitate smooth communication and allows faster emergency response [13].

Stenmark $\lceil 14\rceil$ has developed a model to describe the knowledge management perspective, which is covered by three concepts related to the internet access: information, awareness and communication (see Figure 2 below). These three concepts are directly related to the community as a source of cellular networks and modern smartphones to provide internet access.

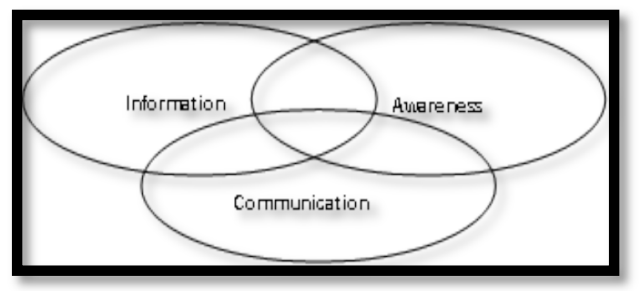

Figure 2. Multiple perspectives on internet.

Mobile phone has become a necessity that should always be present, especially for those living in other parts of the world. As a result, people who are prone to disasters, whether man-made or natural, can now share information using a mobile phone about what is going on.

Increased availability, affordability, infrastructure and the rapid development of mobile services in the mobile telecommunication service subscription opens new opportunities for the dissemination of information, particularly information related to disaster training. Such developments occur at a faster rate in developing countries including rural areas, where communication provides a way to connect with people that cannot be achieved before. The use of smart mobile phones could potentially deliver significant benefits due to the ability to provide and improve access to communications and information resources [13].

United Nations Development Programme $\lceil 15\rceil$ suggested that smart mobile phone, can give opportunities, increasing the productivity for trainees, improve their ability and potential, as well as enable social relationships to strengthen communication in the hierarchy of training and with the community. Organizations are also becoming more effective when the technology have helped people in taking over the job and make the job more quickly and effectively. These infrastructures can be very cost effective. However, the organization will only be more effective, flexible and responsive only if the workforce is highly motivated, well trained and educated [16]

\section{ISSUES DISASTER TRAINING INFORMATON IN MALAYSIA}

The National Security council Directive No. 20 (NSC No. 20): The Policy and Mechanism for National Disaster and Relief Management are the main guidelines for disaster management in Malaysia. The responsibilities and functions of related agencies under an integrated emergency management system are described in the mechanism of the management of disasters.

Disaster management in Malaysia, using ICT, early warnings can be made using sirens, short messaging system (SMS), telephone, telefax, webpage, mass media broadcasting system, and public announcements.The communities and responders are promptly informed and enable them to take necessary actions faster. Besides that, ICT can also be utilized to promote awareness and disseminate early warnings to the public via Fixed-Line Disaster Alert System (FLAS). A separate system known as the Government Integrated Radio Network (GIRN) provides radio communication between responders during an 
emergency or a disaster. Disaster reporting is now more efficient with the centralized Malaysia Emergency Response System (MERS) emergency hotline: 999 [17].

The issues and challenges stated in NSC No. 20 are to enhance institutional capacity and preparedness of agencies in responding to a disaster by conducting more seminars or programs/ drills/ exercises and to increase public awareness, education, and public participation in Disaster Management [18]. Therefore, there are still lack in disseminate information about disaster training to public. It should be given priority to provide awareness to the community at an early stage [1]. However, people are not properly informed about disaster training due to a few reasons:

1) Lack of promotion and exposure about the disaster training information [18] [19][20],

2) Lack of cooperation and collaboration among organizations and NGOs in promoting disaster training

3) Lack of knowledge about disaster preparedness information [21].

4) As everyone knows, disaster training is devoted to flood relief workers and volunteers involved in the disaster. It is open to the public, but due to the lack of information, there is less involvement by the general public.

From the reasons mentioned above, it can be concluded that there is no specific medium available to act as a center to share disaster training information from the training organization to the public. The approach they use is only through the website. Only those who are concerned about disaster training will open the website to find out about the training. But for those who do not care, they will not know about the disaster training information. To overcome this problem, the best approach for a faster information dissemination is by creating a mobile application.

There are many applications have been developed for mobile platforms nowdays [22]. The application is intended to be the center or medium for disaster training information because the organization (the government, non-governmental organizations, and consultants) and community (volunteers and the general public) can obtain and share information [23] with more details about disaster training in Malaysia. Hopefully it can encourage people to participate in the disaster training.

The application is called MyDisasterTraining. The purpose of this application is to provide an opportunity for the organizations to promote disaster training provided for the public in addition to educate the community about the importance of flood training as a preparatory step. In addition, volunteers, aid workers, and the general public can view the training needs. It also encourages cooperation and a partnership information [23], improve understanding, and establish a good relationship with the community, government and training agencies [24]. With this information, the organization will be able to provide the people with the best training based on their needs and requirements.

\section{IV. 'MYDisASTERTRAINING'}

'MyDisasterTraining' application is a prototype mobile application. It is a medium between organizations or indi- vidual and training provider in disseminating disaster training information more effectively (see Figure 3 ).

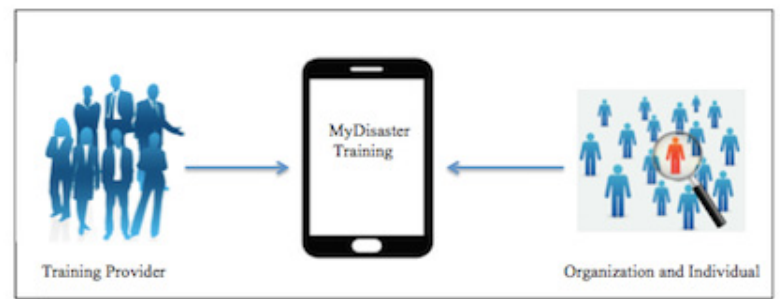

Figure 3. 'MyDisasterTraining' as a third party'

The main goals of the application 'MyDisasterTraining' are:

i) To create a source of specific information on disaster training such as floods

ii) To ensure ongoing development of flood training

iii) The establishment of a series of intergovernmental, volunteer, private and community for the training

The functions of the application'MyDisasterTraining' are:

i) Being the center of training information from multiple organizations in a only single application;

ii) To assist the public to get the latest information on the disaster training provided;

iii) Ensuring effective collaboration between organizations or indvidual and training provider in distribution of knowledge and information on the disaster scenarios.

iv) Maintain a continuous training program in all aspects related to the implementation of a comprehensive flood disaster management to all sectors through institutional and specialist training available.

v) Strengthening the capacity for dissemination of flood disaster management training for each phase of the training.

By creating the application of 'MyDisasterTraining', the expected outcomes are: -

i) An integrated program for comprehensive flood disaster management training at regional and national levels.

ii) An effective mechanism for information dissemination and application of a comprehensive disaster management.

\section{The Flow of Process 'MyDisasterTraining'}

Figure 4 is a flow chart of the mobile application 'MyDisasterTraining' This flow chart is intended to give an overview of the flow process for this application. In this application, there are two types of users, namely the individual (society. volunteers, aid workers) and organizations (government, non-governmental organizations, consultants). Initially, users should download this application. This application is suitable for users who have smartphones. Once users have downloaded this application, the user must $\log$ on as a member by pressing the 'list' and if the user is already registered as a member, they only need to ' $\log$ in'. 
For new users, they need to register and fill out the personal information in advance. User's e-mail is one of the key elements for confirming that the registration is successful. After the user has finished filling out the information needed, press 'send'. After that, a confirmation email will be sent to the e-mail for verification purposes. Links will be provided for verification and after that, the user can log on with the password that has been registered. Similarly, for the newly registered organization, the same steps should be done similar to the new user.

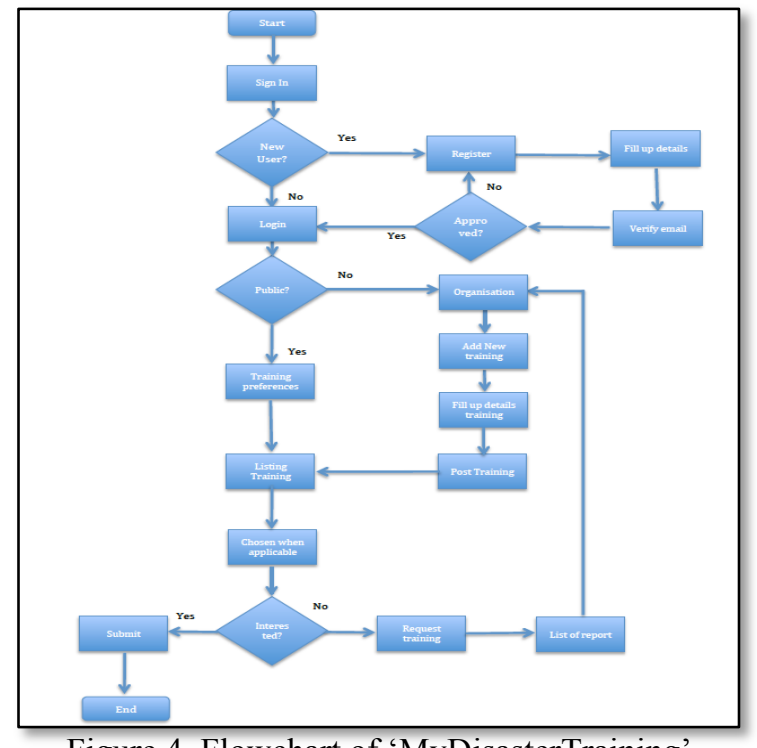

Figure 4. Flowchart of 'MyDisasterTraining'

Users who have registered for the membership applications of 'MyDisasterTraining' can $\log$ in to the application. Then, members are given the option to use the 'search training' at the 'choice of training' to facilitate the search of training required. In this section, there are two 'search tools'; keywords and preferred training location. After filling out the information, members should click 'find'. In this section, the desired results will be displayed. All information is displayed with the details of the training such as venue, date of training, training content, information manager and even a map.

If members are interested in the training that is displayed, they need to press 'apply'. Information will be accepted directly into the organization with the training as requested by members. For members who wish to apply for the training they desire, they need to complete the 'request training' part and fill in the exercises required. This information will be kept as a report and will be posted to the organization for record keeping purposes. Reports of training required by the organization will be a great way to create training that is desired by the members.

Organizations that have registered can promote the training provided by adding new exercises to fill out the information that must be completed, such as training information, date, place and the content of the training. After the organization has successfully completed the form, training will be posted and updated on the list of exercises that will be seen by members.

\section{BENEFITS SMARTPHONE APPLICATION "MYDISASTERTRAINING"}

The advantage of the smart mobile phone application is all of the information are at your fingertips. With a single download of MyDisasterTraining on your smart mobile phone, all information about the training will be updated by the organizations involved. People can decide whether the exercise provided will help to improve their skills and knowledge or not [25]. Apart from that, the use of smart mobile phone application is also inexpensive and can be accessed anytime and anywhere. Furthermore, rural communities can also get disaster training information quickly and effectively.

Other benefits of using mobile technology include access to the right training at the right time and improve internal communications within a complex training system. More effective designs which focus on technology, ease of use [26], and coupled with the dissemination of training in using modern tools [27] are among the advantages of a mobile application. In addition, this application provides an opportunity for the community to recommend any exercises that may be appropriate for their skills and knowledge. It also gives the people freedom to share ideas and opinions from the community's point of view to the organizations in order to provide a training that can benefit the community in the future. This application will have a significant impact among the communities and the organizations with the creation of useful exercises which are beneficial for both sides.

\section{RECOMMENDATION}

To promote a wider use of this application, the use of MyDisasterTraining shall involve a variety of agencies in Malaysia in the future. The joint venture, which includes the government, volunteers, and rescue agencies, will give great benefits to the organizers of the flood disaster training program, in order to ensure a more effective program [28]. Elements of Islamic knowledge are also good to be added for future improvements of the mobile application, especially for the Muslim society, for example, how to pray during a disaster.

\section{CONCLUSION}

MyDisasterTraining is one of the smart mobile phone prototype applications which act as a center for information about disaster training in Malaysia. The apps involve the government sectors, NGOs, private sectors, and other agencies. It is hoped that this application can increase public awareness, education level, and public participation in disaster management, as hoped by the government. By using smart mobile phones, the information regarding disaster training will be disseminated continuously, immediately, and efficiently, and the training can be carried out effectively. The impact from this application are Malaysians will get accurate and up-to-date disaster training information and at the same time help them to increase their knowledge and skills about disaster preparedness before the real disaster happens.

\section{REFERENCES}

[1] Aini, M. S., Ibrahim, W. M. N., Daud, M., Fakhru'l-Razi, A., \& Tangavelu, S. K., "Study on emergency response preparedness of hazardous materials transportation", Disaster Prevention and Management, vol. 20, pp. 266-280, 2001. http://dx.doi.org/10.1108/09653560110395331 
[2] International Federation of Red Cross and Red Cresent Societies, A Practical Guide to: Gender-sensitive Approaches for Disaster 2011 , available

https://www.ifrc.org/PageFiles/96532/A\%20Guide\%20for\%20Ge nder-sensitive\%20approach\%20to\%20DM.pdf

[3] International Federation of Red Cross and Red Cresent Societies, Introduction to Disaster Preparedness: Disaster Preparedness in Training Programme, 2000, available at http://www.ifrc.org/Global/Introdp.pdf

[4] Peters, K., Learning on the move: Mobile technologies in business and education, 2005, available at http://pre2005.flexiblelearning.net.au/projects/resources/2005/Lear ning\%20on $\% 20$ the $\% 20$ move final.pdf.

[5] Kattan, Ali., Abdullah, Rosni., Abdul Salam, Rosalina., \& Ramedas, Sureswaran, "Building Distributed Heterogeneous Smart Phone Java Applications an Evaluation from a Development Perspective", Journal of ICT, vol. 8, pp. 67-83, 2009.

[6] Hussain, Azham., Hashim, Nor Laily., Nordin, Nazib., \& Tahir, Hatim Mohamad, "A Metric -Based Evaluation Model for Applications on Mobile Phones", Journal of ICT, vol. 12, pp. 5571, 2013.

[7] El-Hussein, M. O. M., \& Cronje, J. C., "Defining mobile learning in the higher education landscape", Educational Technology \& Society, vol. 13, pp. 12-21, 2010.

[8] Jeng, Y., Wu, T., Huang, Y., Tan, Q., \& Yang, S., "The add-on impact of mobile applications in learning strategies: A review study", Educational Technology \& Society, vol. 13, pp. 3-11, 2010

[9] Kerrigan, Karen \& Keating, Raymond J., Saving time \& Money with mobile applications: a small business 'apps'ortunity, 2011, available

http://www.sbecouncil.org/uploads/Mobile\%20APP\%20Final\%20 Report\%20SBE\%20Council.pdf

[10] Belisario JS, Marcano, Huckvale K., Greenfield G., Car J., \& Glun LH., Smartphone \& Tablet self management apps for asthma (Review), 2013, available at http//www.thecochranelibrary.com

[11] Knuth, R., "So vereignty, Globalism, and Information Flow in Complex Emergencies", The Information Society, vol. 15, pp. 1119, 1999. http://dx.doi.org/10.1080/019722499128637

[12] Al-Mahadeen, B.M., "Factors Affecting the Readiness of Medical Doctors and PAtients with Chronic Conditions toward the Usage of Smartphones in the Saudi Arabian Healthcare Sector", International Journal of Interactive Mobile Technologies, vol. 9, no. 1, pp. 47-52, 2015. http://dx.doi.org/10.3991/ijim.v9i1.4279

[13] Chib, Arul., "The Aceh Besar midwives with mobile phones project: Design and evaluation perspectives using the information and communication technologies for healthcare development model", Journal of Computed-Mediated Communication, vol. 15, pp. 500-525, 2010. $\quad$ http://dx.doi.org/10.1111/j.10836101.2010.01515.x

[14] Stenmark, D., "Information vs. knowledge: the role of intranets in knowledge management", In Proceedings of the 35th Hawaii International Conference on Systems Sciences, 2002. http://dx.doi.org/10.1109/HICSS.2002.994043

[15] United Nations Development Programme. (2005). Promoting ICT for human development in Asia: Realizing the millennium development goals. India: Elsevier.

[16] Wills, Mike., "Managing the Training Process: putting the basics into practice", Journal of European Industrial Training, vol. 18, pp. 4-28, 1994. http://dx.doi.org/10.1108/03090599410062507

[17] Buleting MERS 999, 2013, available at http://www1.999.gov.my/ admin999/downloads/MERS_buletin_3 .pdf
[18] Rosli, Ruhizal \& O'Brien, Geoff. "Social learning in managing disasters in Malaysia", Journal of Disaster Prevention and Management, vol. 20, pp. 386-397, 2011. http://dx.doi.org/10.1108/09653561111161716

[19] Engelman, A., Ivey, S. L., Tseng, W., Dahrouge, D., Brune, J., \& Neuhauser, L., "Responding to the deaf in disasters: establishing the need for systematic training for state-level emergency management agencies and community organizations", BMC health services research, vol. 13, no. 84, 2013. http://dx.doi.org/10.1186/1472-6963-13-84

[20] History, D. T., "Introduction to International Disaster Management: The Management of Disasters", pp. 1-35, 2007.

[21] Leonard H. Guercia, J., "Integration of Training Civilian and Military Disaster Responders", 2011, available at https://www.hsdl.org/?view\&did=691401

[22] Algahtani, M. \& Fayyoumi, A., "Mobile Application Development for Quran Verse Recognition and Interpretations", International Journal of Interactive Mobile Technologies, vol. 9, no. 1, pp. 1922, 2015. http://dx.doi.org/10.3991/iijim.v9i1.4171

[23] Yap, Nonita T., Disaster Management Developing Country Communities \& Climate Change: The Role of ICTs, University of Manchester, 2011.

[24] Munodawafa, Jacob., "Role of Telecommunication \& ICTs in Disaster Management", In workshop of ITU Southern \& East Africa on the Use of Telecommunication/ICT for Disaster Management: savings life Southern Africa, 2008.

[25] Pawar, Manohar., "Disaster Preparedness/Management Agencies \& Centers", Asia Pacific Journal of Social Work \& Development, vol. $18, \quad$ pp. 84-91, 2012. http://dx.doi.org/10.1080/216 50993.2008.9756045

[26] Venkatesh, V., \& Morris, M. G., "Why don’t men ever stop to ask for directions? Gender, social influence, and their role in technology acceptance and usage behavior", MIS Quarterly, vol. 24, pp. 115-139, 2000. http://dx.doi.org/10.2307/3250981

[27] Ganapathy, K., \& Ravindra, A., "mHealth: A potential tool for health care delivery in India", In Proceeding of the Making the eHealth Connection: Global Partnerships, Local Solutions Conference Bellagio, Italy, 2008.

[28] Mahmud, A. R., Shattri, M., Ghazali, A. H., \& Billa, L., "Comprehensive planning and the role of SDSS in flood disaster management in Malaysia," Disaster Prevention and Management, vol. 15, pp. 233-240, 2006. http://dx.doi.org/10.1108/096535606 10659775

\section{AUTHORS}

Nik Nadian Nisa Nik Nazli is a PhD Student at Universiti Sains Islam Malaysia (USIM), Malaysia (e-mail: nadian_nisa13@yahoo.com).

Sapora Sipon is with Universiti Sains Islam Malaysia (USIM). She is now with the Faculty of Leadership and Management, USIM (e-mail: sapora@usim.edu.my).

Norita Md Norwawi is with Universiti Sains Islam Malaysia (USIM). She is now with the Faculty of Science and Technology, USIM (e-mail: norita@usim.edu.my).

This research is conducted with the grant awarded by the Ministry of Higher Education Malaysia (USIM/LRGS(UUM)/FKP/ULUM/34/ 50112) entitled "Flood Management Impact Reduction and Relief: Flood Relief Management, A Psychosocial Dynamic Model for Well Being". Submitted 27 January 2015. Published as resubmitted by the authors 20 August 2015. 